Proceedings of the 1998 IEEE/RSJ

Intl. Conference on Intelligent Robots and Systems

Victoria, B.C., Canada • October 1998

\title{
On Covariances for fusing Laser Rangers and Vision with Sensors Onboard a Moving Robot
}

\author{
Jonas Nygårds ${ }^{1}$ and Åke Wernersson 2,1 \\ 1) Robotics / Autonomous Mechanical Systems, IKP, Linköping University, \\ S-58183 Linköping, Sweden, jonny@ikp.liu.se, robotics@ikp.liu.se \\ 2) Robotics \& Automation, Luleå University of Technology, \\ S-97187 Luleå, Sweden, akewe@sm.luth.se, robotics@sm.luth.se
}

\begin{abstract}
Consider a robot to measure or operate on man made objects randomly located in the workspace. The optronic sensing onboard the robot are a scanning range measuring time-of-flight laser and a CCD camera. The goal of this paper is to give explicit covariance matrices for the extracted geometric primitives in the surrounding workspace. Emphasis is on correlation properties of the stochastic error models during motion. Topics studied includes;

- Covariance of Radon/Hough peaks for plane surfaces

- Covariance's for the intersection of two planes

- Equations for combining vision features, plane surfaces and range discontinuities.

- Explicit equations of how the covariance matrices are transformed during the robot motion.

Typical applications are; Models for verification and updating of CAD-models when navigating inside buildings and industrial plants. Accumulating sensor readings for a telecommanded robot.

Keywords: Optronic Sensors, Range Laser, Camera, Radon / Hough transform, Estimation of Planes, Estimation of Corners, Primitives in CAD-models, Navigation.
\end{abstract}

\section{Introduction}

Figures 2-4 are laser measurements and a CCD image from a fairly uncluttered indoor scene. The goal of this paper is not algorithms for extracting the geometric primitives but rather explicit covariance matrices for describing the uncertainty. The estimated primitives should both be used for controlling the robot, for sensor allocation and also allowing new measurements after motion of the robot to be fused in a consistent way.

Related work on navigation of mobile robots based on plane surfaces are found in, say; [Cro89], [HBC96], [LFW96], [MoC89] and [SSC90] while [KLO93], presents a non parametric solution for natural environments. Special references to covariances for estimating planes are found in [Kan94] and [Lar95].

The explicit equations for the covariances of the estimated features are approximations based on models for sensor resolution, number of measurements in a sector etc. The coordinate systems

$0-7803-4465-0 / 98 \$ 10.00$ (c) 1998 IEEE are selected by geometry and the resulting covariances outlines the nature of the uncertainty in the estimates of planes and their intersections.

The result complement earlier results on stochastic maps [MoC89], ,[SSC90] and related work as [Tar92] towards an important class of applications. The motivation behind the work is the need for explicit models in design of feedback control and sensor planning as in [NNW97] and [NiN96].

\section{Covariance Matrix for the Extracted Radon Parameters of a Plane}

Consider $\mathrm{N}$ range measurements on a segment of a plane. In this section a covariance matrix for the

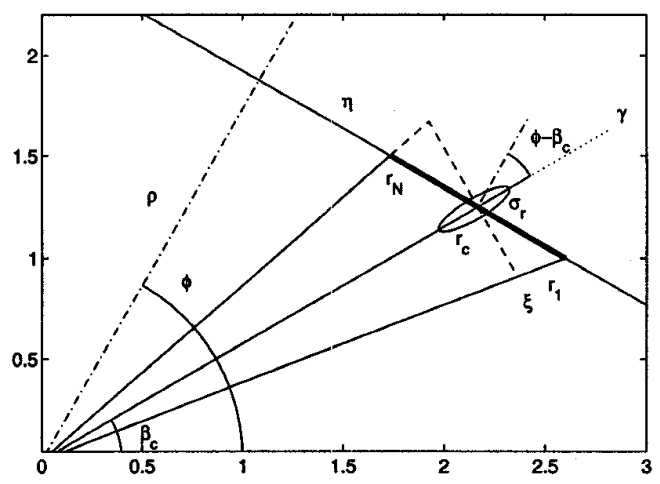

Figure 1 A vertical plane parametrized by the range $\rho$ and the perpendicular orientation $\phi$. On a segment of the plane $N$ range measurements are measured. An approximation of the covariance matrix for $(\rho, \phi)$ is given below

extracted parameters describing the plane is derived. First consider a vertical plane described by the distance $\rho$ to the sensor and the orientation $\phi$ of the perpendicular. On a segment of the vertical plane a set of ranges $r_{I} \ldots r_{N}$ are measured with a constant step $\delta_{\beta}$ in angle. These $\mathrm{N}$ measurements gives a Center of Gravity (COG) described in polar co-ordinates as $\left(r_{c}, \beta_{c}\right)$, compare this with Figure 1. 

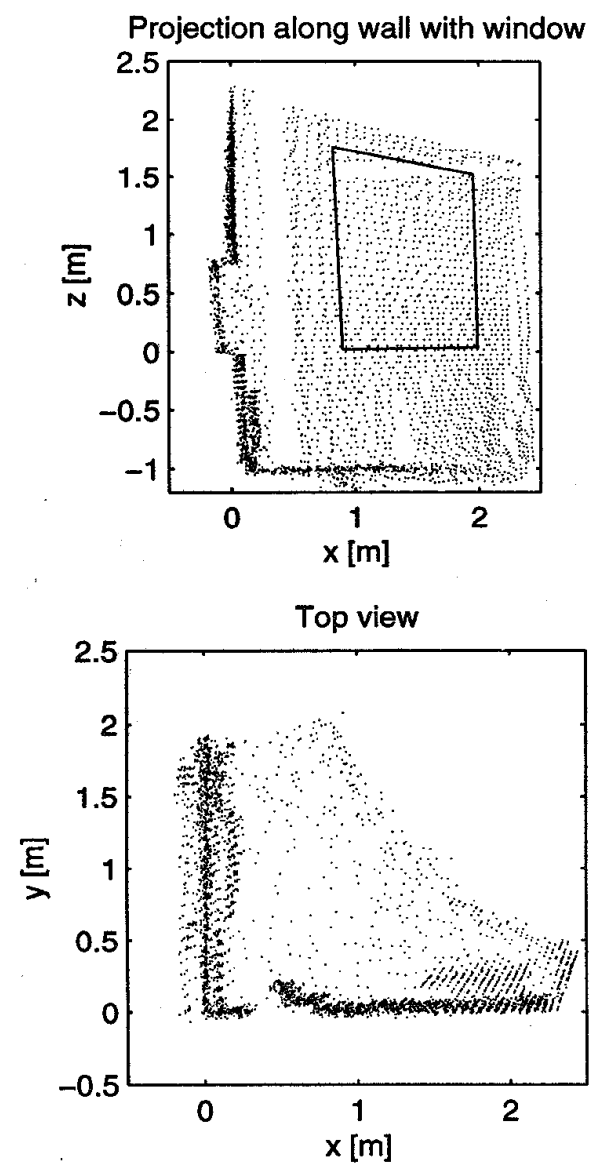

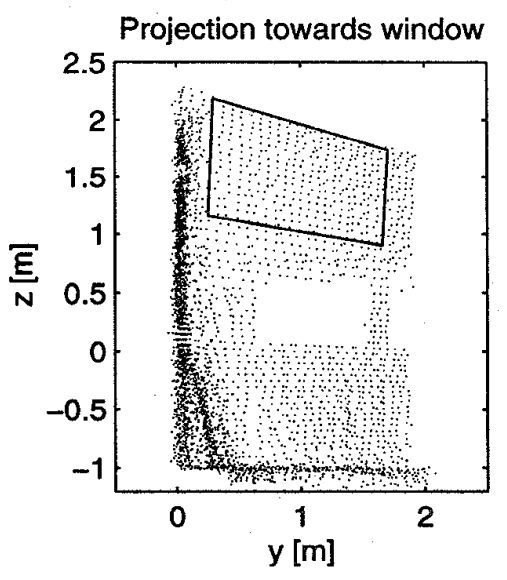

3D-view from outside looking in

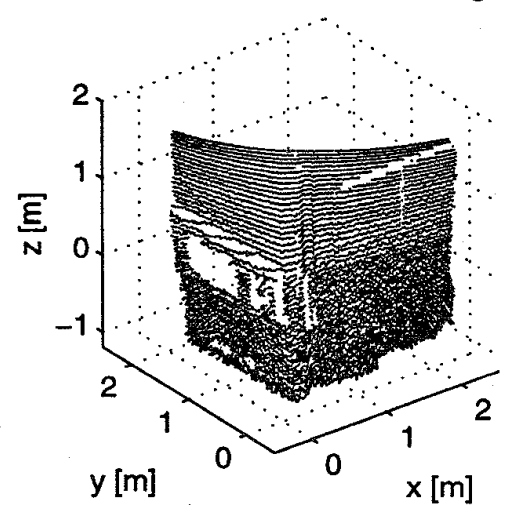

Figure 2 Three projections and a $3 D$-view of range data from a ladar scan of the corner in the CCD image of Figure 3. The data has been transformed to a co-ordinate frame where the line of intersection between the two walls is the z-axis The $x$-axis is given as the normal of the plane corresponding to the trapezoid above the window. Note that the wall is not flat! - the planes above and under the window have a mutual difference of $2-5 \mathrm{~cm}$. Several objects are easily identified like the radiator below the window, an I-beam at $x=.5 \mathrm{~m}, y=.1 \mathrm{~m}$ and $a$ pipe at $x=.3$ $m, y=.2 m$. At $x=1.2-2 m$ there is a concrete slab and a couple of stones leaning against the wall.

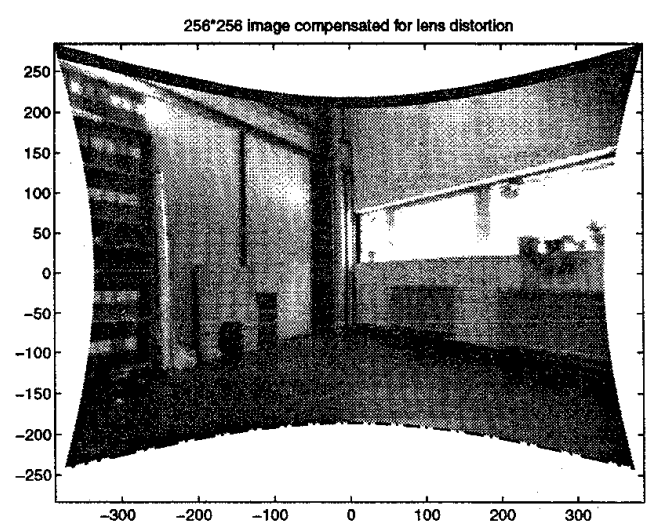

Figure 3 Wide angle CCD-image of a corner in the laboratory. The heat radiators, the I-beam and pipe in the corner and some flowers in the window are clearly visible. To the left is a slab of concrete leaning towards to the wall. The image is partially compensated for radial distortion.

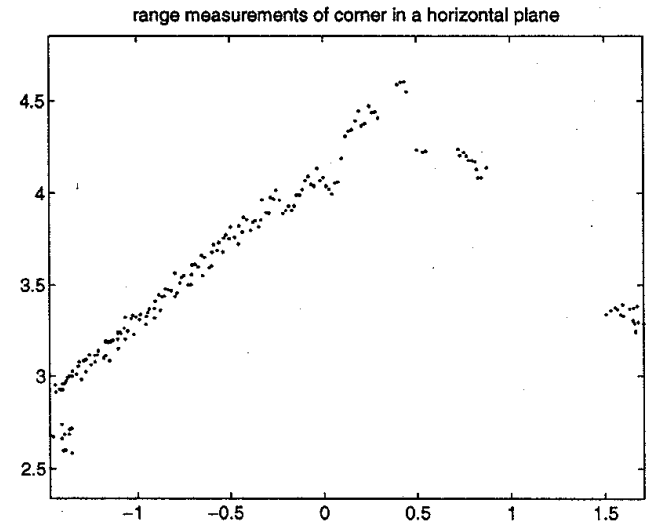

Figure 4 A horizontal range slice from the range measuring laser. The pipe and I-beam in the corner are detected as well as the flowers. The window does not give any return to the laser. The bookshelf to the left is barely visible. 
Next, drop a local Cartesian coordinate system $(\xi, \gamma)$ at the COG. Each measurement can then be written

$$
\gamma_{i}=k\left(\xi_{i}-\bar{\xi}\right)+\bar{\gamma}+\varepsilon_{i}
$$

where $\varepsilon_{i}$ is the range noise with variance $\sigma_{r}^{2}$. In this equation $\xi_{n} \approx r_{c} \delta_{\beta}\left(n-\frac{N}{2}\right)$ and $\gamma_{i} \approx r_{i}-r_{c}$

Using linear regression gives the estimate

$$
\begin{gathered}
\hat{k}=\frac{\sum_{i=1 . N}\left(\gamma_{i}-\bar{\gamma}\right)\left(\xi_{i}-\bar{\xi}\right)}{\sum_{i=1 . N}\left(\xi_{i}-\bar{\xi}\right)^{2}} \\
\operatorname{Var}(k) \approx \frac{\sigma_{r}^{2}}{\sum_{i=1}^{N}\left(\xi_{i}-\bar{\xi}\right)^{2}} \approx \\
\frac{\sigma_{r}^{2}}{\sum_{i=1}^{N}\left(r_{c}^{2} \delta_{\beta}^{2} i-r_{c}^{2} \delta_{\beta}^{2} \frac{N+1}{2}\right)^{2}} \approx \frac{12 \sigma_{r}^{2}}{r_{c}^{2} \delta_{\beta}^{2} N^{3}}
\end{gathered}
$$

From Figure 1, estimates of the $(\rho, \phi)$ representation of the line follows as

$$
\begin{aligned}
& \hat{\rho}=r_{c} \cos (\arctan (\hat{k})) \\
& \hat{\phi}=\beta_{c}+\arctan (\hat{k})
\end{aligned}
$$

Ignoring the uncertainty in $\beta$ gives

$$
\sigma_{\phi}^{2}=\operatorname{Var}(\phi) \approx \frac{12 \sigma_{r}^{2}}{\left(r \delta_{\beta}\right)^{2} N^{3}} \frac{1}{\left(1+\hat{k}^{2}\right)^{2}} \underset{\text { small } \hat{k}}{\approx} \frac{12 \sigma_{r}^{2}}{\left(r \delta_{\beta}\right)^{2} N^{3}}
$$

Introducing

$$
\sigma_{\rho c}^{2}=\cos ^{2}(\arctan (\hat{k})) \frac{\sigma_{r}^{2}}{N}
$$

the error covariance can be written

$$
\operatorname{Cov}\left(\left[\begin{array}{ll}
\rho & \phi
\end{array}\right]^{T}\right) \approx\left(\begin{array}{cc}
\sigma_{\rho c}^{2}+\hat{\eta}^{2} \sigma_{\phi}^{2} & \hat{\eta} \sigma_{\phi}^{2} \\
\hat{\eta} \sigma_{\phi}^{2} & \sigma_{\phi}^{2}
\end{array}\right)
$$

where $\hat{\eta}=-r_{c} \sin (\arctan (\hat{k}))$ is the distance between the COG and the closest point on the plane. Now if the robot moves the orientation uncertainty should to still pivot around the COG when the old measurements are compared with the new.

Interpretation: $A$ robot travelling along the surface at a constant distance keeps $(\hat{\rho}, \hat{\phi})$ constant but the covariance matrix will change due to changing $\eta$

In the regression analysis the uncertainty in the direction $\beta$ of the range samples is not included.

\section{Fisher Information Matrix for the Extracted Radon Parameters of a Plane}

Below we are to describe the estimates using the information matrix in the sense of Fisher i. e. the inverse of the covariance matrix. First consider a plane parmetrized as before by $u=[\rho, \phi]$ and the distance function:

$$
F(z, u)=x \cos (\phi)+y \sin (\phi)-\rho=0
$$

A single measurement on this plane is

$$
z_{i}=\left[x_{i}, y_{i}\right]+\varepsilon_{i}
$$

where the noise is modelled as $E\left[\varepsilon_{i}\right]=0 \quad E\left[\varepsilon_{i} \varepsilon_{i}^{T}\right]=R$

The information matrix from a single measurement is then approximated to the first order by the matrix:

$$
\begin{aligned}
& \frac{\partial F(z, u)}{\partial u}\left(\frac{\partial F(z, u)}{\partial z} \operatorname{cov}(z)\left(\frac{\partial F(z, u)}{\partial z}\right)^{T}\right)^{-1}\left(\frac{\partial F(z, u)}{\partial u}\right)^{T}= \\
& {[-1-x \sin (\phi)+y \cos (\phi)]\left(\sigma_{f}^{2}\right)^{-1}[-1-x \sin (\phi)+y \cos (\phi)]^{T}} \\
& =\frac{1}{\sigma_{f}^{2}}\left[\begin{array}{cc}
1 & -\eta \\
-\eta & \eta^{2}
\end{array}\right] \\
& \eta=-x \sin (\phi)+y \cos (\phi) \\
& \sigma_{f}^{2}=\left[\begin{array}{ll}
\cos (\phi) & \sin (\phi)] R[\cos (\phi) \\
\sin (\phi)
\end{array}\right]
\end{aligned}
$$

are used.

The geometric interpretations of $\eta$ and $\sigma_{f}^{2}$ are

$\eta=$ distance from the COG to the perpendicular of the plane.

$\sigma_{f}^{2}=$ projection of the measurement noise covariance on

the normal direction of the plane $i$. e. the direction where the noise "contributes".

The resulting information matrix of the $\mathrm{N}$ measurements is then:

$$
\sum_{i=1}^{N} \frac{1}{\sigma_{f i}^{2}}\left[\begin{array}{cc}
1 & -\eta_{i} \\
-\eta_{i} & \eta_{i}^{2}
\end{array}\right]_{\sigma_{A}^{2}=\text { const. }}^{\approx} \frac{1}{\sigma_{f}^{2}}\left[\begin{array}{cc}
N & -N \bar{\eta} \\
-N \bar{\eta} & \sum \eta_{i}^{2}
\end{array}\right]
$$

Comparing with the inverse of (7) gives

$$
\begin{aligned}
& \left(\begin{array}{cc}
\sigma_{\rho c}^{2}+\hat{\eta}^{2} \sigma_{\phi}^{2} & \hat{\eta} \sigma_{\phi}^{2} \\
\hat{\eta} \sigma_{\phi}^{2} & \sigma_{\phi}^{2}
\end{array}\right)^{-1}=\left(\begin{array}{cc}
\frac{1}{\sigma_{\rho c}^{2}} & -\frac{\hat{\eta}}{\sigma_{\rho c}^{2}} \\
-\frac{\hat{\eta}}{\sigma_{\rho c}^{2}} & \frac{\sigma_{\rho c}^{2}+\hat{\eta}^{2} \sigma_{\phi}^{2}}{\sigma_{\rho c}^{2} \sigma_{\phi}^{2}}
\end{array}\right) \approx \\
& \frac{1}{\cos ^{2}(\phi) \sigma_{r}^{2}}\left(\begin{array}{c}
N \\
-N \hat{\eta}
\end{array}\left(\frac{\left(r_{c} \delta_{\beta}\right)^{2} N(N-1)(N+1)}{12}+N \hat{\eta}^{2}\right)\right)
\end{aligned}
$$

For 3D measurements $z_{i}=\left[x_{i}, y_{i}, z_{i}\right]+\varepsilon_{i}$ the plane is parmetrised by $u=[\rho, \theta, \phi]$. Introduce the distance function:

$$
\begin{aligned}
& F(\mathbf{z}, \mathbf{u})= \\
& x \cos (\phi) \cos (\theta)+y \sin (\phi) \cos (\theta)-z \sin (\theta)-\rho=0
\end{aligned}
$$

The corresponding information turn out to be 


$$
\sum_{i=1}^{N} \frac{1}{\sigma_{f i}^{2}}\left[\begin{array}{ccc}
1 & \xi_{i} & -\eta_{i} \cos (\theta) \\
\xi_{i} & \xi_{i}^{2} & -\xi_{i} \eta_{i} \cos (\theta) \\
-\eta_{i} \cos (\theta) & -\xi_{i} \eta_{i} \cos (\theta) & \eta_{i}^{2} \cos ^{2}(\theta)
\end{array}\right]
$$

where the $(\eta, \xi)$ paremetrisation is

$$
\begin{aligned}
& \eta=-x \sin (\phi)+y \cos (\phi) \\
& \xi=x \cos (\phi) \sin (\theta)+y \sin (\phi) \sin (\theta)+z \cos (\theta)
\end{aligned}
$$

and the projected noise is

$$
\begin{aligned}
\sigma_{f}^{2}= & {\left[\begin{array}{lll}
\cos (\phi) \cos (\theta) & \sin (\phi) \cos (\theta) & -\sin (\theta)
\end{array}\right]^{*} } \\
& R\left[\begin{array}{lll}
\cos (\phi) \cos (\theta) & \sin (\phi) \cos (\theta) & -\sin (\theta)
\end{array}\right]^{T}
\end{aligned}
$$

\section{The Covariance Matrix for an Intersecting 'Vision Plane'}

For convenience consider the intersection of two planes in Figure 5, expressed in the co-ordinate system attached to plane 1 i. e. Plane 1 constrain the intersection to lie along the $\eta$-direction:

$$
\begin{aligned}
& \boldsymbol{x}_{1 i}=\left[\begin{array}{lll}
0 & \eta_{1 i} & 0
\end{array}\right] \\
& C\left(\boldsymbol{x}_{1 i}\right)=\left[\begin{array}{ccc}
\sigma_{\rho 1}{ }^{2}+\eta_{1 i}^{2} \sigma_{\phi 1}^{2} & 0 & \eta_{1 i} \sigma_{\phi 1}^{2} \\
0 & s & 0 \\
\eta_{1 i} \sigma_{\phi 1}^{2} & 0 & \sigma_{\phi 1}^{2}
\end{array}\right]
\end{aligned}
$$

Since $\eta_{l i}$ is unknown $s$ should be considered as very large i.e. $1 / \mathrm{s} \rightarrow 0$. Now express the intersection as a point on plane 2 relative to the local co-ordinate system at plane 1 :

$$
\begin{aligned}
\mathbf{x}_{2 i}= & \mathbf{R}\left(\phi_{1}-\phi_{2}\right)\left[\begin{array}{lll}
\rho_{21} & \eta_{2 i} & 0
\end{array}\right] \\
C\left(\mathbf{x}_{2 i}\right)= & \mathbf{R}\left(\phi_{1}-\phi_{2}\right)\left[\begin{array}{ccc}
\sigma_{\rho 2}{ }^{2}+\eta_{2 i}^{2} \sigma_{\phi 2}^{2} & 0 & \eta_{2 i} \sigma_{\phi 2}^{2} \\
0 & s & 0 \\
\eta_{2 i} \sigma_{\phi 2}^{2} & 0 & \sigma_{\phi 2}^{2}
\end{array}\right] \\
& \times \mathbf{R}\left(\phi_{1}-\phi_{2}\right)^{T}+C\left(\mathbf{x}_{1}\right)+M C\left(\mathbf{x}_{1}\right) M^{T}
\end{aligned}
$$

where $\boldsymbol{R}(\phi)$ is the rotation matrix around the vertical and $M$ is the magnification matrix due to the orientation uncertainty, [D-W88], given by:

$$
\begin{aligned}
\boldsymbol{R}(\phi) & =\left[\begin{array}{ccc}
\cos (\phi) & \sin (\phi) & 0 \\
-\sin (\phi) & \cos (\phi) & 0 \\
0 & 0 & 1
\end{array}\right] \\
M & =\left[\begin{array}{ccc}
0 & 0 & -\eta_{21} \\
0 & 0 & \rho_{21} \\
0 & 0 & 0
\end{array}\right]
\end{aligned}
$$

The two expressions for the intersection can now be fused using a Kalman filter:

$$
\boldsymbol{x}_{i}=(\boldsymbol{I}-\boldsymbol{K}) \boldsymbol{x}_{1 i}+\boldsymbol{K} \boldsymbol{x}_{2 i}
$$

using the Kalman gain [BSL93 p.139]

$$
\begin{aligned}
& K=C\left(x_{i}\right) C\left(x_{i 2}\right)^{-1} \\
& =\left[\begin{array}{ccc}
0 & 0 & 0 \\
-\cot \left(\phi_{1}-\phi_{2}\right) & 1 & 0 \\
0 & 0 & 1
\end{array}\right]
\end{aligned}
$$

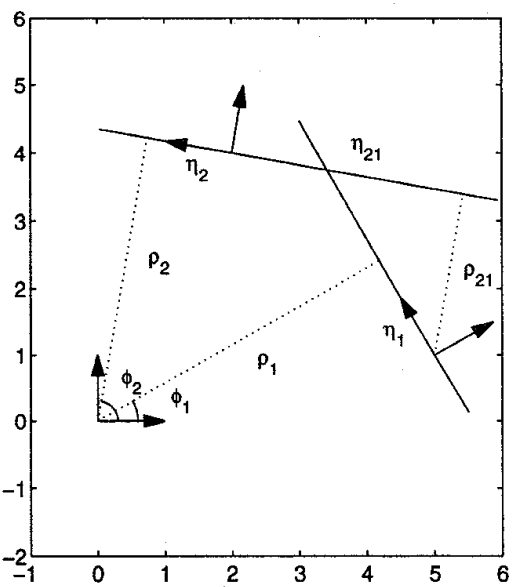

Figure 5 The intersection between two planes where plane $I$ could be the plane of an edge detected in a camera and plane 2 estimated from range measurements.

$$
\begin{aligned}
& C\left(\mathbf{x}_{i}\right)=\left(C\left(\mathbf{x}_{1 i}\right)^{-1}+C\left(\mathbf{x}_{2 i}\right)^{-1}\right)^{-1}= \\
& {\left[\begin{array}{ccc}
\sigma_{a}^{2} & \sigma_{a}^{2} \cot \left(\phi_{1}-\phi_{2}\right) & 0 \\
\sigma_{a}{ }^{2} \cot \left(\phi_{1}-\phi_{2}\right) & \frac{\sigma_{b}{ }^{2}+\sigma_{a}^{2} \cos ^{2}\left(\phi_{1}-\phi_{2}\right)}{\sin ^{2}\left(\phi_{1}-\phi_{2}\right)} & 0 \\
0 & 0 & \sigma_{\phi 1}^{2}
\end{array}\right]}
\end{aligned}
$$

where $\sigma_{a}{ }^{2}$ and $\sigma_{b}{ }^{2}$ are the [1,1] elements of the covariance matrices $C\left(x_{i 1}\right), C\left(x_{i 2}\right)$ respectively.

$$
\begin{aligned}
\sigma_{a}^{2}= & \cos ^{2}\left(\arctan \left(\hat{k}_{1}\right)\right) \frac{\sigma_{r}^{2}}{N_{1}}+\hat{\eta}_{1 i}{ }^{2} \frac{12 \sigma_{r}^{2}}{r_{1}^{2} \delta_{\beta}^{2} N_{1}{ }^{3}} \\
\sigma_{b}^{2}= & \cos ^{2}\left(\arctan \left(\hat{k}_{2}\right)\right) \frac{\sigma_{r}^{2}}{N_{2}}+\hat{\eta}_{2 i}{ }^{2} \frac{12 \sigma_{r}^{2}}{r_{2}^{2} \delta_{\beta}^{2} N_{2}{ }^{3}} \\
+ & \sigma_{a}^{2} \cos ^{2}\left(\phi_{1}-\phi_{2}\right)+\sigma_{\phi 1}^{2}\left(2 \eta_{1} \cos \left(\phi_{1}-\phi_{2}\right)\right. \\
& \times\left(\rho_{21} \sin \left(\phi_{1}-\phi_{2}\right)+\eta_{21} \cos \left(\phi_{1}-\phi_{2}\right)\right) \\
& \left.+\left(\eta_{21} \cos \left(\phi_{1}-\phi_{2}\right)+\rho_{21} \sin \left(\phi_{1}-\phi_{2}\right)\right)^{2}\right)
\end{aligned}
$$

Note that in the calculation of the inner inverses in (18) the limit for $1 / \mathrm{s} \rightarrow 0$ is used.

The transformation from the co-ordinates of plane 1 to world co-ordinates is performed using the formalism presented by Smith, Self and Cheeseman in [SSC90] (cp. the transformation in eq. (16)).

The generalisation for $3 D$ is to transform the problem to a co-ordinate system with a $\mathrm{z}$-coordinate direction parallel to the direction of the intersection. If the 
direction of the two planes is represented by normal vectors the direction of the new $\mathrm{z}$-axis is:

$$
e_{z}=\frac{n_{1} \times n_{2}}{\left|n_{1} \times n_{2}\right|}
$$

In this coordinate system the solution for the intersection in eq. (15)-(19) can be used. Given perpendicular distance estimates $\hat{\rho}_{1}, \hat{\rho}_{2}$ and the estimated direction vectors $\hat{n}_{1}, \hat{n}_{2}$ for the two intersecting planes a more general formula for the point on the line with position vector perpendicular to the line is:

$$
\begin{aligned}
& {\left[\begin{array}{l}
x_{3} \\
y_{3} \\
z_{3}
\end{array}\right]=\frac{\left(\hat{\rho}_{1}-\hat{\rho}_{2}\left(\hat{n}_{1} \bullet \hat{n}_{2}\right)\right)}{1-\left(\hat{n}_{1} \bullet \hat{n}_{2}\right)^{2}} \hat{n}_{1}+} \\
& +\frac{\left(\hat{\rho}_{2}-\hat{\rho}_{1}\left(\hat{n}_{1} \bullet \hat{n}_{2}\right)\right)}{1-\left(\hat{n}_{1} \bullet \hat{n}_{2}\right)^{2}} \hat{n}_{2}
\end{aligned}
$$

As reference frame orientation one of the planes normals is used as $\mathrm{x}$-direction and the normalised cross product of the normals as $z$. i.e. the reference frame of the corner is:

$$
R_{3}=\left[\begin{array}{c}
\hat{n}_{1}^{T} \\
\left(\left(\hat{n}_{1} \times \hat{n}_{2}\right) /\left\|\hat{n}_{1} \times \hat{n}_{2}\right\| \times \hat{n}_{1}\right)^{T} \\
\left(\hat{n}_{1} \times \hat{n}_{2}\right)^{T} /\left\|\hat{n}_{1} \times \hat{n}_{2}\right\|
\end{array}\right]=\left[\begin{array}{c}
\boldsymbol{e}_{x} \\
\boldsymbol{e}_{y} \\
\boldsymbol{e}_{z}
\end{array}\right]
$$

Following Kanatani [Kan94] a first order approximation of the covariance of a normalised vector is:

$$
\begin{aligned}
& \text { If } n_{i}=\frac{a}{\|a\|} \text { then } \\
& C\left(n_{i}\right) \approx \frac{1}{\|a\|^{2}}\left(I-n_{i} n_{i}{ }^{T}\right) C(a)\left(I-n_{i} n_{i}{ }^{T}\right)
\end{aligned}
$$

With resulting covariance for the direction along the intersection (assuming independence between plane estimates)

$$
\begin{aligned}
& C\left(e_{z}\right)=C\left(\frac{\hat{n}_{1} \times \hat{n}_{2}}{\left\|\hat{n}_{1} \times \hat{n}_{2}\right\|}\right)= \\
& \frac{P_{z}\left(\tilde{n}_{2} C\left(\hat{n}_{1}\right) \tilde{n}_{2}+\tilde{n}_{1} C\left(\hat{n}_{2}\right) \tilde{n}_{1}\right) P_{z}}{\left\|\hat{n}_{1} \times \hat{n}_{2}\right\|^{2}}
\end{aligned}
$$

where the projection matrix is

$$
P_{z}=\left(I-e_{z} e_{z}^{T}\right)
$$

and the tilde $(\sim)$ denotes the skew symmetric matrix corresponding to a vector cross product i.e.

$$
\begin{aligned}
& \tilde{n}_{1} n_{2}=n_{1} \times n_{2} \\
& \tilde{n}_{1}=\left[\begin{array}{ccc}
0 & -n_{z_{1}} & n_{1} \\
n_{z_{1}} & 0 & -n_{1} \\
-n_{1} & n_{1} & 0
\end{array}\right]
\end{aligned}
$$

Observe also that $\left\|\hat{n}_{1} \times \hat{n}_{2}\right\|^{2}=1-\left(\hat{n}_{1} \circ \hat{n}_{2}\right)^{2} \equiv s_{\alpha}^{2}$.
The covariances and cross covariances with the other direction vectors are:

$$
\begin{aligned}
& C\left(e_{x}\right)=C\left(\hat{n}_{1}\right) \\
& C\left(e_{y}\right)=P_{y}\left(\tilde{e}_{z} C\left(\hat{n}_{1}\right) \tilde{e}_{z}^{T}+\tilde{n}_{1} C\left(e_{z}\right) \tilde{n}_{1}^{T}-\right. \\
& \left.\frac{\tilde{n}_{1} \tilde{n}_{2} C\left(\hat{n}_{1}\right) \tilde{e}_{z}^{T}+\tilde{e}_{z} C\left(\hat{n}_{1}\right)\left(\tilde{n}_{1} \tilde{n}_{2}\right)^{T}}{s_{\alpha}}\right) P_{y} \\
& C\left(\boldsymbol{e}_{x}, e_{y}\right)=P_{x}\left(C\left(\hat{n}_{1}\right) \tilde{e}_{z}^{T}-C\left(\hat{n}_{1}\right)\left(\tilde{n}_{1} \tilde{n}_{2}\right)^{T} / s_{\alpha}\right) P_{y} \\
& C\left(e_{x}, e_{z}\right)=\frac{P_{x} C\left(\hat{n}_{1}\right) \tilde{n}_{2}^{T} P_{z}}{s_{\alpha}} \\
& C\left(e_{y}, e_{z}\right)=P_{y}\left(\tilde{e}_{z} C\left(\hat{n}_{1}\right) \tilde{n}_{2}^{T} / s_{\alpha}-\tilde{n}_{1} C\left(e_{z}\right)\right) P_{z}
\end{aligned}
$$

\section{An example of uncertainty for two intersecting planes}

We conclude by an example illustrating the double coned shape of the uncertainty. For simplicity, the intersection of the two planes defines the $\mathrm{z}$-axis, see Figure 6. Also, let plane 1 have it's normal in the direction of the $x$-axis. The angle between the planes is $\phi_{2}$.

The laser is sampling in a rectangular grid with spacing $\Delta$ on each plane as illustrated in Figure 6. For plane 1 the $m_{1} \times n_{1}$ range measurements are:

$$
Z_{1}=\left\{\left[\begin{array}{c}
0 \\
\Delta\left(i-\frac{m_{1}+1}{2}\right)+\eta_{1} \\
\Delta\left(j-\frac{n_{1}+1}{2}\right)+\xi_{1}
\end{array}\right]+v_{i j}, i=1 . . m_{1}, j=1 . . n_{1}\right\}
$$

where the range noise is independent in the grid with

$$
E\left[v_{i j}\right]=\mathbf{0}, E\left[v_{i j} v_{i j}^{T}\right]=\sigma_{r}^{2}
$$

Plane 2 has angle $\phi_{2}$ from the x-axis. The $m_{2} \times n_{2}$ measurements on plane 2 are (expressed in a co-ordinate frame oriented along the normal of plane 2 ):

$$
Z_{2}=\left\{\left[\begin{array}{c}
0 \\
\Delta\left(i-\frac{m_{2}+1}{2}\right)+\eta_{2} \\
\Delta\left(j-\frac{n_{2}+1}{2}\right)+\xi_{2}
\end{array}\right]+w_{i j}, i=1 . . m_{2}, j=1 . . n_{2}\right\}
$$

where

$$
E\left[w_{i j}\right]=0, E\left[w_{i j} w_{i j}^{r}\right]=\sigma_{r}^{2}
$$




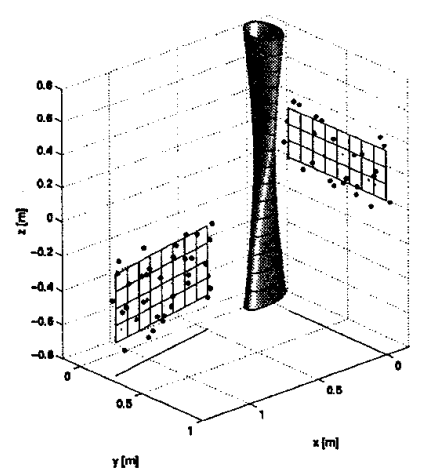

Figure 6 The intersection between measured planes where the grids represents the measurement site. The twisted tubular surface represents $3 \sigma$ uncertainties for the line of intersection between the two planes. Finally the points represents the measurements with $\sigma_{r}=0.033 m, \Delta=0.1 m$.

For this selected case the information matrices for the two planes becomes

$$
\begin{gathered}
\Gamma_{1}=\frac{m_{1} n_{1}}{\sigma_{r}^{2}}\left[\begin{array}{ccc}
1 & \xi_{1} & -\eta_{1} \\
\xi_{1} & \xi_{1}^{2}+\frac{\Delta^{2}\left(n_{1}^{2}-1\right)}{12} & -\xi_{1} \eta_{1} \\
-\eta_{1} & -\xi_{1} \eta_{1} & \eta_{1}^{2}+\frac{\Delta^{2}\left(m_{1}^{2}-1\right)}{12}
\end{array}\right] \\
\Gamma_{2}=\frac{m_{2} n_{2}}{\sigma_{r}^{2}}\left[\begin{array}{ccc}
1 & \xi_{2} & -\eta_{2} \\
\xi_{2} & \xi_{2}^{2}+\frac{\Delta^{2}\left(n_{2}^{2}-1\right)}{12} & -\xi_{2} \eta_{2} \\
-\eta_{2} & -\xi_{2} \eta_{2} & \eta_{2}^{2}+\frac{\Delta^{2}\left(m_{2}^{2}-1\right)}{12}
\end{array}\right]
\end{gathered}
$$

where the last matrix is expressed in a co-ordinate frame oriented as plane 2 .

For each of the planes the corresponding covariance matrices are

$$
\operatorname{cov}([\rho, \theta, \phi])=\left[\begin{array}{ccc}
\sigma_{c}^{2}+\sigma_{\phi}^{2} \eta^{2}+\sigma_{\theta}^{2} \xi^{2} & -\xi \sigma_{\theta}^{2} & \eta \sigma_{\phi}^{2} \\
-\xi \sigma_{\theta}^{2} & \sigma_{\theta}^{2} & 0 \\
\eta \sigma_{\phi}^{2} & 0 & \sigma_{\phi}^{2}
\end{array}\right]
$$

where $\sigma_{c}^{2}=\frac{\sigma_{r}^{2}}{m n}$

$$
\sigma_{\theta}^{2}=\frac{12 \sigma_{c}^{2}}{\Delta^{2}\left(n^{2}-1\right)} \quad \sigma_{\phi}^{2}=\frac{12 \sigma_{c}^{2}}{\Delta^{2}\left(m^{2}-1\right)}
$$

The estimate of the $x-y$ coordinates for the intersection has, using (18), the covariance matrix:

$$
\begin{aligned}
& C\left(\boldsymbol{x}_{i}\right)=\left(C\left(\boldsymbol{x}_{1 i}\right)^{-1}+C\left(\boldsymbol{x}_{2 i}\right)^{-1}\right) \\
& =\left[\begin{array}{cc}
\sigma_{a}^{2} & \sigma_{a}^{2} \cot \left(\phi_{2}\right) \\
\sigma_{a}^{2} \cot \left(\phi_{2}\right) & \frac{\sigma_{a}^{2} \cos ^{2}\left(\phi_{2}\right)+\sigma_{b}^{2}}{\sin ^{2}\left(\phi_{2}\right)}
\end{array}\right]
\end{aligned}
$$

where

$$
\begin{aligned}
& \sigma_{a}^{2}=\frac{\sigma_{r}^{2}}{m_{1} n_{1}}\left(1+\eta_{1}^{2} \frac{12}{\Delta^{2}\left(m_{1}^{2}-1\right)}+\xi_{1}^{2} \frac{12}{\Delta^{2}\left(n_{1}^{2}-1\right)}\right) \\
& \sigma_{b}^{2}=\frac{\sigma_{r}^{2}}{m_{2} n_{2}}\left(1+\eta_{2}^{2} \frac{12}{\Delta^{2}\left(m_{2}^{2}-1\right)}+\xi_{2}^{2} \frac{12}{\Delta^{2}\left(n_{2}^{2}-1\right)}\right)
\end{aligned}
$$

The surface representing the uncertainty of the line of intersection in Figure 6 was calculated using equation (31) in a reference plane of varying height i.e. for varying $\xi_{1}, \xi_{2}$.

The covariances for the direction estimates, as expressed in a coordinate system oriented as plane1 are: Plane 1

$$
\operatorname{cov}\left(n_{1}\right)=\left[\begin{array}{ccc}
0 & 0 & 0 \\
0 & \sigma_{\phi 1}^{2} & 0 \\
0 & 0 & \sigma_{\theta 1}^{2}
\end{array}\right]
$$

with $\sigma_{c 1}^{2}=\sigma_{r}^{2} / m_{1} n_{1}$ and

$$
\sigma_{\theta 1}^{2}=\frac{12 \sigma_{c 1}^{2}}{\Delta^{2}\left(n_{1}^{2}-1\right)} \quad \sigma_{\phi 1}^{2}=\frac{12 \sigma_{c 1}^{2}}{\Delta^{2}\left(m_{1}^{2}-1\right)} .
$$

Plane 2

$$
\begin{aligned}
& \operatorname{cov}\left(n_{2}\right)= \\
& {\left[\begin{array}{ccc}
\sin \left(\phi_{2}\right)^{2} \sigma_{\phi 2}^{2} & -\sin \left(\phi_{2}\right) \cos \left(\phi_{2}\right) \sigma_{\phi 2}^{2} & 0 \\
-\sin \left(\phi_{2}\right) \cos \left(\phi_{2}\right) \sigma_{\phi 2}^{2} & \cos \left(\phi_{2}\right)^{2} \sigma_{\phi 2}^{2} & 0 \\
0 & 0 & \sigma_{\theta 2}^{2}
\end{array}\right]}
\end{aligned}
$$

with $\sigma_{c 2}^{2}=\sigma_{r}^{2} / m_{2} n_{2}$ and

$$
\sigma_{\theta 2}^{2}=\frac{12 \sigma_{c 2}^{2}}{\Delta^{2}\left(n_{2}{ }^{2}-1\right)} \quad \sigma_{\phi 2}^{2}=\frac{12 \sigma_{c 2}^{2}}{\Delta^{2}\left(m_{2}{ }^{2}-1\right)}
$$

Using equation (25) the covariance for the direction of the intersection then follows as:

$$
\begin{aligned}
& C\left(\mathbf{e}_{z}\right)=C\left(\left(\hat{\mathbf{n}}_{1} \times \hat{\mathbf{n}}_{2}\right) /\left\|\hat{\mathbf{n}}_{1} \times \hat{\mathbf{n}}_{2}\right\|\right)= \\
& \frac{\left(I-\mathbf{e}_{\mathbf{z}} \mathbf{e}_{\mathbf{z}}^{\mathbf{T}}\right)\left(\widetilde{\mathbf{n}}_{2} C\left(\hat{\mathbf{n}}_{1}\right) \widetilde{\mathbf{n}}_{2}+\widetilde{\mathbf{n}}_{1} C\left(\hat{\mathbf{n}}_{2}\right) \tilde{\mathbf{n}}_{1}\right)\left(I-\mathbf{e}_{\mathbf{z}} \mathbf{e}_{\mathbf{z}}^{\mathbf{T}}\right)}{\sin \left(\phi_{2}\right)^{2}}
\end{aligned}
$$

i.e.

$$
C\left(e_{z}\right)=\left[\begin{array}{ccc}
\sigma_{\theta 1}^{2} & -\sigma_{\theta 1}^{2} \cot \left(\phi_{2}\right) & 0 \\
-\sigma_{\theta 1}^{2} \cos \left(\phi_{2}\right) & \frac{\sigma_{\theta 2}^{2}+\sigma_{\theta 1}^{2} \cos \left(\phi_{2}\right)^{2}}{\sin \left(\phi_{2}\right)^{2}} & 0 \\
0 & 0 & 0
\end{array}\right]
$$

Using [SSC90], the example and the results above can be transformed to other co-ordinate systems. 


\section{Conclusions and future work}

Navigation based on stochastic modelling and estimated line / surface segments from range data has proven successful in many cases. An explicit expression of the first order approximation of the covariance for the Hough / Radon line parameters $\left[\begin{array}{ll}\rho & \phi\end{array}\right]^{T}$ is

$$
C\left(\left[\begin{array}{ll}
\rho & \phi
\end{array}\right]^{T}\right) \approx\left[\begin{array}{cc}
\sigma_{\rho}^{2}+\eta^{2} \sigma_{\phi}^{2} & \eta \sigma_{\phi}^{2} \\
\eta \sigma_{\phi}^{2} & \sigma_{\phi}^{2}
\end{array}\right]
$$

where $\rho=$ perpendicular distance, $\eta=$ distance from normal to COG along the line and $\phi=$ the orientation of the line normal. An important result concerning the representation of lines and planes measured from a moving platform is that the "centre of gravity" for old measurements "follow along with the robot as it moves". This is expressed by how $\eta$ varies as the robot moves.

Using the covariance for separate lines the expression for the covariance of the intersection was also presented. The results are easily generalised to apply to planes and their intersections. Also, the results are directly applicable to range cameras, provided the range dependent resolution is introduced, i. e. $\sigma_{r} \approx$ constant $R^{2} \sigma_{0}$

With a good representation of the covariance for intersections of planes the fusion of laser range measurements and edges detected in a CCD image can be performed. The intersection of the infinite plane represented by the $\mathrm{CCD}$ edge and planes detected in laser data are combined. The same applies for a corner generated by two planes.

In the present work no attention has been paid to the problem of segmentation and association. However, a good representation of uncertainty is a prerequisite for good associations and one natural extension of the work is to consider the association problem using a Gaussian Mixture setting [NNW96],[BSL93 pp.461-465].

\section{References}

[BSL93] Bar-Shalom, Y. and Li, X.-R. Estimation and Tracking: Principles, Techniques, and Software, Artech House, 1993.

[Cro89] Crowley, J. (1989). World Modelling and Position Estimation for a Mobile Robot Using Ultrasonic Ranging, IEEE Int. Conf Robotics and Automation, 1989, pp 674-680.

[D-W88] Durrant-Whyte, H.F., Uncertain Geometry, IEEE Journal of Robotics \& Automation Vol 4, 1988

[HBC96] Hebért, P. Betgé-Brezetz and Chatila, R. Decoupling Odometry And Exteroceptive Perception In Building A Global World Map of a Mobile Robot: The Use of Local Maps, Proc. IEEE Int. Conf Robotics and Automation, 1996, Minnesota, April 1996, pp 757-764
[Kan94] Kanatani, K. "Statistical analysis of geometric computation" CVGIP: Image Understanding, Vol.59, No.3, pp. 286-306, 1994

[KLO93] P. Klöör, P. Lundquist, P. Ohlsson, Å. Wernersson, Change Detection ,IFAC Intelligent Autonomous Vehicles, Southampton, May 93, pp 7176

[Lar95] U. Larsson, Mobile Robot Localisation using Electro-Optical Sensors, Lic. thesis, (HLU-TH-L-1995/42-L--SE) Dec. 1995

[LFW96] U. Larsson, J. Forsberg, §. Wernersson "Mobile Robot Localisation: Integrating Measurements from a Time-of-Flight Laser", IEEE Transactions on Industrial Electronics, special issue on Multi Sensor Fusion and Integration for Intelligent Systems, Vol. 43, No. 3, June 1996. pp. 422-432.

[Tar92] Tardós, J., D. Representing partial and uncertain sensorial information using the theory of symmetries. Proc. IEEE Int. Conf Robotics and Automation, pp1799-1804, Nice, France, 1992

[LFW96] U. Larsson, J. Forsberg, ^. Wernersson "Mobile Robot Localisation: Integrating Measurements from a Time-of-Flight Laser", IEEE Transactions on Industrial Electronics, special issue on MultiSensor Fusion and Integration for Intelligent Systems, Vol. 43, No. 3, June 1996. pp. 422-432.

[MoC89] Moutarlier, P. and Chatila, R. (1989) An Experimental System for Incremental Environment Modelling by an Autonomous Mobile Robot, 1st Int. Symp. on Experimental Robotics, Montreal, 1989, Springer Verlag.

[NiN96] Nilsson B. and Nygårds J. Sensor Motion Planning with Active Uncertainty Reduction: Gripping and Docking Tasks, Proc. IEEE Int. Conf. on Robotics and Automation, Vol. 4 pp. 3026-3033, Minneapolis, April 22-28,1996.

[NNW96] Nilsson, B., Nygårds, J. and Wernersson, $\AA$., On $2 D$ Posture Estimation with Large Initial Orientation Uncertainty and Range Dependant Noise, Int. Conf. on Automation Robotics and Computer Vision, Singapore 1996

[NNW97] Nilsson B., Nygårds J. and Wernersson $\AA$., "On-Range Sensor Feedback for Mobile Robot Docking Within Prescribed Posture Tolerances.", Journal of Robotic Systems Vol. 14 No. 4, pp. 297312, John Wiley \& Sons, Inc., 1997.

[SSC90] Smith, R., Self, M. and Cheeseman, P. "Estimating Uncertain Spatial Relationships in Robotics," Autonomous Robot Vehicles, SpringerVerlag, pp 167-193. 1990 